\title{
MICROFLUIDIC DETECTION AND ANALYSIS BY INTEGRATION OF EVANESCENT WAVE SENSING WITH THERMOCAPILLARY ACTUATION \\ J. P. Valentino ${ }^{l}$, S. M. Troian ${ }^{2}$, and S. Wagner ${ }^{l}$ \\ ${ }^{1}$ Department of Electrical Engineering, Princeton University, Princeton, NJ, USA \\ ${ }^{2}$ Department of Chemical Engineering, Princeton University, Princeton, NJ, USA
}

\begin{abstract}
An integrated system capable of microfluidic actuation, detection and sensing is described which combines evanescent wave sensing with thermocapillary manipulation. Liquid droplets or streams transported across the beam path of a planar thin film waveguide, which encapsulates the microheater array, induce attenuation of the propagating waveguide modes. The attenuated signal is used to monitor droplet location, dye concentration in aqueous solutions and reaction kinetics for enzymatic hydrolysis of the sugar X-galactose by beta-galactosidase.
\end{abstract}

\section{INTRODUCTION}

Current development of various surface tension-driven microfluidic devices [1-5] requires new and innovative methods for in-situ droplet detection and analysis. Given that portability is a major design goal for most microfluidic devices, such sensors should be directly incorporated into the chip to minimize size requirements. Sensor integration can also provide additional benefits such as reducing fabrication and packaging costs.

The location, volume and composition of droplets can be detected in a number of ways. For thermocapillary actuation by embedded microheater arrays, the resistor rise time upon activation can be used to extract droplet volume, given the thermal diffusivity of the overlying liquid sample [1]. Non-uniform heating, however, can cause undesired liquid migration away from the sensing regions by thermocapillary forces. A second method relies on miniaturized coplanar capacitive sensors responsive to changes in the dielectric constant of the overlying liquid medium [6,7]. These sensors, however, can be hampered by large mutual capacitive effects and charge buildup within the droplet. Planar optical waveguides, by contrast, offer the possibility of non-intrusive detection and analysis. The main challenge, however, rests with direct integration of the optical coupling elements and planar waveguide with the device elements used for fluidic transport and mixing. The design described provides high sensitivity and minimal additional fabrication and design steps for open format microfluidic devices.

Previous work on integration of evanescent wave sensing with liquid diagnostics has focused primarily on enclosed flow cells or enclosed microfluidic channels [8-11]. In these studies, the liquid sample is positioned to flow continuously over the entire surface of the guided beam. For open microfluidic devices based on droplet manipulation, sample detection requires closed loop control of droplet position as well as composition. While other groups have demonstrated the use of planar waveguides to launch and collect light across enclosed fluidic channels $[12,13]$, the device layouts are not easily adaptable to open format chips. We present in this paper the design and fabrication of a planar optical waveguide coupled with a surface tension driven microfluidic system that can detect droplet location, measure sample concentration, and monitor on-chip droplet reactions.

\section{THEORETICAL BACKGROUND}

\section{Integrated Optical Waveguide Sensors}

For the planar waveguide shown in Fig. 1 and $n_{\text {liquid }}=n_{l}$, light propagates by total internal reflection in an optically dense thin film of refractive index $n_{2}$ situated in-between a supporting substrate and a sample (superstrate) of lower refractive indices, $\boldsymbol{n}_{3}$ and $\boldsymbol{n}_{l}$, respectively. Light can only propagate at specific angles $\theta$ corresponding to solutions of the eigenvalue equation [14]

$K_{2} d-\tan ^{-1}\left(\frac{K_{1}}{K_{2}}\right)-\tan ^{-1}\left(\frac{K_{3}}{K_{2}}\right)=m \pi$

where $d$ is the waveguide thickness and $m=0,1,2 \ldots$ is the mode number. For $p$-polarized light (i.e. transverse magnetic [TM] modes) of wavelength $\lambda$,

$$
K_{i}^{T M}=\left(\frac{2 \pi}{\lambda n_{i}^{2}}\right) \sqrt{\left|\left(n_{2} \sin \theta\right)^{2}-n_{i}^{2}\right|} .
$$

The reflections at each interface give rise to an electric field, known as an evanescent wave, whose amplitude decays exponentially with distance within the lower refractive index medium. The propagation length is normally confined to distances much less than $100 \mathrm{~nm}$. Analyte concentration in the superstrate can therefore be measured by monitoring absorption of the evanescent wave energy. The absorbance $A$ is given by

$A=\varepsilon c N L$

where $\varepsilon$ is the sample's molar absorptivity, $c$ is the molarity, $N$ is the total number of reflections at the waveguide/superstrate interface, and $L$ is the effective evanescent path length. Both $N$ and $L$ are a function of waveguide refractive index as well as mode number and their product yields a useful comparison to absorbance measurements by conventional spectrophotometers. In addition to absorption, the signal output can also be modulated by changes in the superstrate refractive index, as for example in Fig. 1, where an overlying droplet covers a portion of the waveguide surface with a different refractive index, $n_{\text {liquid. }}$ The discontinuity in the superstrate refractive 


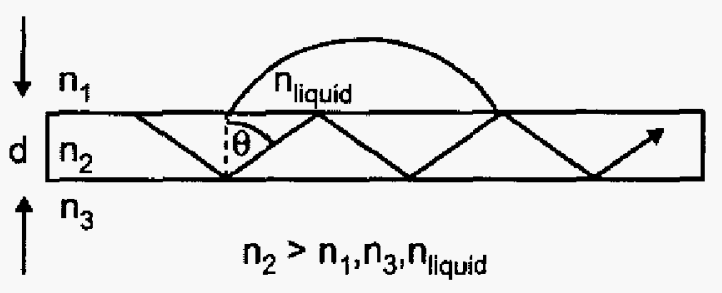

Figure 1: Illustration of total internal reflection in thin film planar waveguide with guiding angle $\theta$.

index leads to reflective losses of the incident light as well as attenuation of the higher order modes [15].

\section{Thermocapillary Flow}

The droplets on the chip surface are transported using thermocapillary flow $[1,2]$. This actuation method uses a series of embedded thin film resistors to create a gradient in surface tension across the droplet surface by locally heating the trailing end. The induced thermocapillary shear stress, $\tau=(\partial \gamma / \partial T) \nabla T$ (where $T$ is the local temperature), pulls the droplet away from the heated regions toward cooler portions of the pathway. The liquid is confined laterally from uncontrolled spreading by lithographically patterning the chip surface with perfluorinated silanized selfassembling monolayers into hydrophobic and hydrophilic regions. For the studies presented here, the droplet height to length ratio was approximately 0.05 , and the droplets were transported by thermal gradients of approximately $7^{\circ} \mathrm{C} / \mathrm{mm}$.

\section{DESIGN AND FABRICATION}

Figure 2 shows the device cross-section and experimental setup. The supporting substrate consisted of a $0.7 \mathrm{~mm}$ thick Corning 1737 glass slide. Thin-film titanium heaters (50 $\mathrm{nm}$ ) having a length, width and spacing equal to $0.8 \mathrm{~mm}, 3$ $\mathrm{mm}$, and $60 \mu \mathrm{m}$, respectively, were patterned on the glass surface by electron beam evaporation. A $2 \mu \mathrm{m}$ Cyclotene ${ }^{\mathrm{TM}}$ $B C B$ polymer $(n=1.56)$ was then spun onto the surface and cured at $250^{\circ} \mathrm{C}$ for 2 hours. The $\mathrm{BCB}$ polymer is used to planarize the device surface in order to reduce waveguide scattering and absorption caused by the non-uniform surface with discrete heaters. A SiN $\mathrm{N}_{\mathrm{x}}$ waveguiding layer $(800 \mathrm{~nm}, n$ $=1.83$ ) was then deposited using plasma enhanced chemical vapor deposition. Finally, the waveguide surface was treated with a hydrophobic monolayer and patterned to create $2 \mathrm{~mm}$ wide hydrophilic pathways for lateral confinement of droplets $[1,2]$.

The fabricated chip was placed on an $x-y$ microscope translation stage. A pair of in-coupling and out-coupling prisms (SFL57 glass, $\mathbf{n}=1.84$ ) was secured to the waveguide surface by applying pressure to their top faces

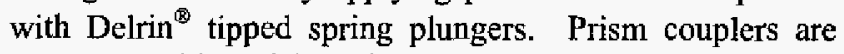
more amenable to lab trials than grating couplers in that no fabrication is required and coupling efficiency is typically $50 \%$ or higher [16]. The waveguide parameters for the sensor (effective refractive index, number of modes, and (a)

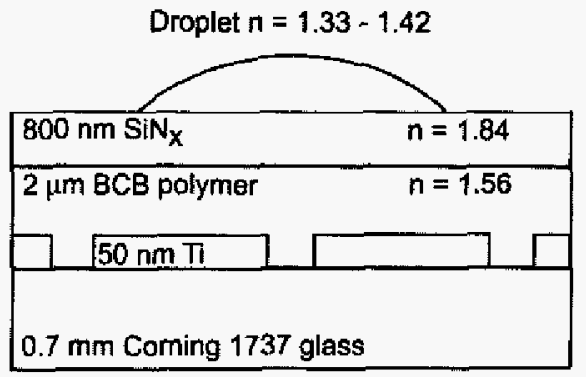

(b)

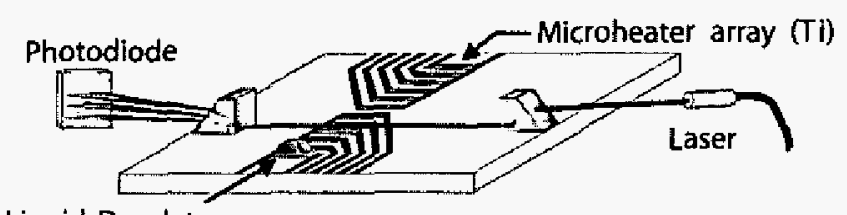
Liquid Droplet

Figure 2: (a) Device cross-section (not to scale). (b) Experimental setup: HeNe laser light is prism-coupled into and out of $S_{i N}$, film of microfluidic device; photodiode output used to monitor changes in attenuation as droplet traverses optical beam path.

incident angle for each mode) were determined via the prism coupling method [17]. For this device, three modes of propagation are allowed. TM-potarized $632.8 \mathrm{~nm} \mathrm{He}-\mathrm{Ne}$ laser light was then coupled into a single mode polarizationpreserving fiber that was mounted to a goniometer stage capable of vertical and horizontal translation. The stage was rotated to ensure that the light from the fiber was incident on the prism face at an angle equal to the coupling angle of the $\mathrm{m}=1$ mode of the waveguide. Due to scattering, some of the coupled light also propagates in the $\mathrm{m}=0$ and 2 modes. The decoupled output was then measured via a photodetector at the face of the out-coupling prism. A lockin amplifier was used to display the output voltage, which is directly proportional to the detected photocurrent.

\section{RESULTS AND DISCUSSION}

Figure 3 shows the photodiode response of an experiment performed for two separate $500 \mathrm{nl}$ droplets of dodecane. The first droplet was electronically programmed to pass over the guided beam at a speed of $250 \mu \mathrm{m} / \mathrm{s}$. Detection of the droplet is indicated by the sharp dip in voltage. The second drop was then moved into position directly above the beam, held in place for three minutes by thermocapillary trapping [2], and finally transported away. As evident in the figure, the guided beam intensity dropped as the liquid was positioned over the beam path, remained at this lower level for three minutes and then almost completely resumed its baseline value.

Using the BeamPROP waveguide modeling software, we attempted to simulate the attenuation effects caused by a rectangular liquid element located over a guided beam with similar aspect ratios, waveguide thickness and refractive indices as in the experiment. Figure 4 shows an example of the simulated waveguide output power normalized to the 


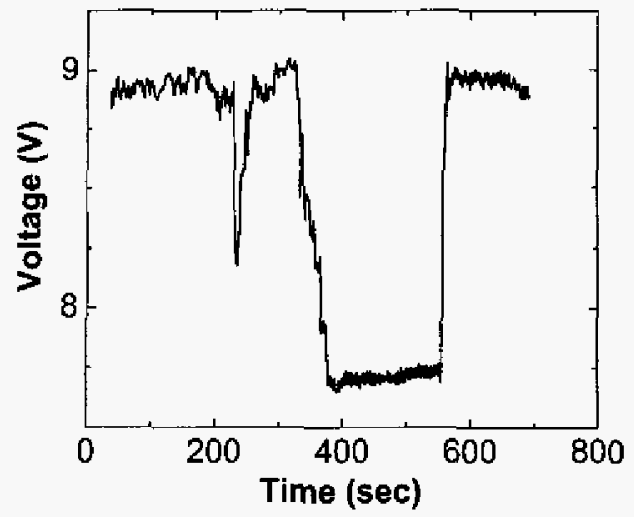

Figure 3: Photodiode response to traversal of two $500 \mathrm{nl}$ droplets of dodecane across beam path. The first droplet passes at $250 \mu \mathrm{m} / \mathrm{s}$ while the second droplet is held for 3 minutes over the beam and then moved away.

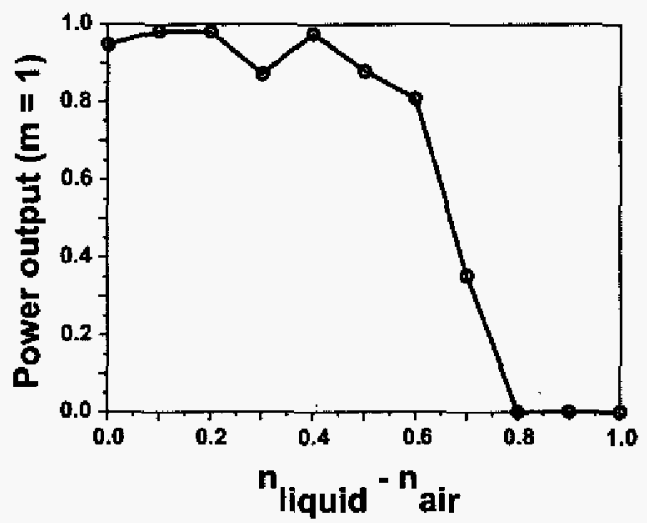

Figure 4: Normalized power output for $m=1$ mode vs. refractive index difference for $2 \mathrm{~mm}$ wide droplet $\left(n_{a t r}=1\right)$.

launched power for the $m=1$ mode. The refractive index of the sample liquid element was varied from 1 to 2 in steps of 0.1 . As seen in Fig. 4, the attenuation caused by a modification of the superstrate refractive index is negligible in the range of interest, i.e. $1.30-1.45$. These simulations therefore suggest that other mechanisms, like scattering losses due to the wedge profile at the droplet edges, are responsible for the large (up to $30 \%$ ) attenuation ratios obtained in experiment. Different wedge profiles would also account for the discrepancy in maximum attenuation obtained for each droplet as seen in Fig. 3.

To demonstrate the use of evanescent sensing for liquid concentration measurements, $400 \mathrm{nl}$ droplets of aqueous solutions of FD\&C Blue 1 dye (molar absorptivity $=1.38 \mathrm{x}$ $10^{-5} \mathrm{M}^{-1} \mathrm{~cm}^{-1}$ ) were transported across the beam path at a speed of $250 \mu \mathrm{m} / \mathrm{s}$. The absorption of evanescent wave energy as the droplet traverses the beam is directly proportional to the dye concentration. The normalized voltage response, $\mathrm{V} / \mathrm{V}_{0}$, for droplets of different dye concentration is plotted in Fig. 5 , where $V$ is the photodiode voltage output as the droplet is transported past the beam

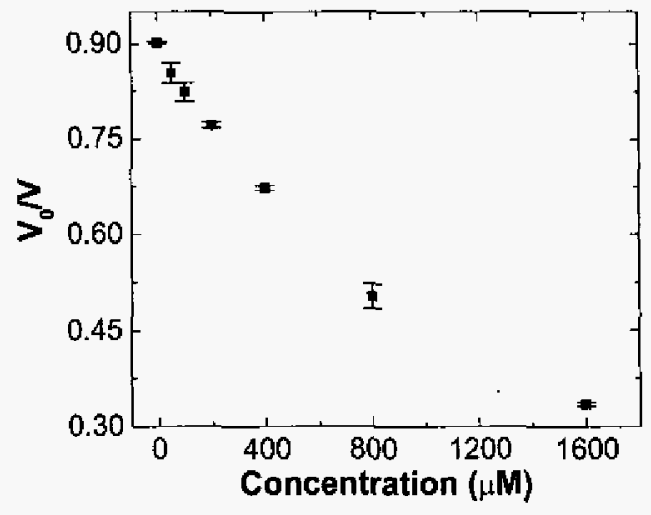

Figure 5: Absorbance response of sensor vs. concentration for water droplets containing the dye FD\&C Blue 1. Each data point is an average of three measurements.

and $V_{0}$ is the reference voltage measured with no liquid present. The response can be increased by coupling to a higher order mode as well as by optimizing the waveguide thickness and refractive index. These studies indicate that the dye does not adhere to the waveguide surface since the signal output is observed to recover its baseline value after the droplet has passed. The limit of detection of concentration was calculated using three times the root mean square of signal noise and in this case was equivalent to about $3.8 \mu \mathrm{M}$.

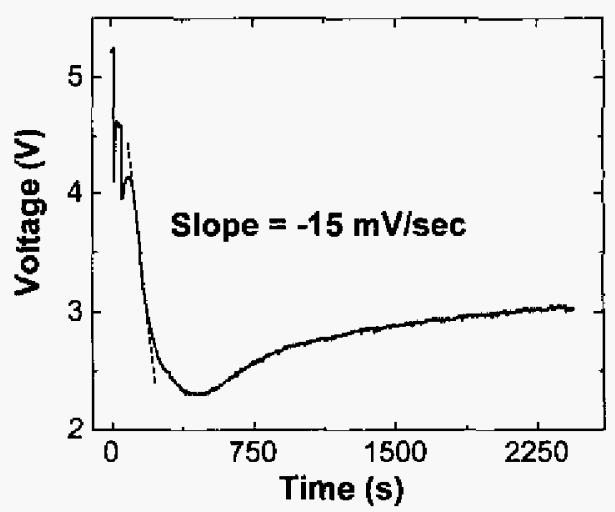

Figure 6: Sensor response to enzymatic breakdown of $X$-gal at $T=22{ }^{\circ} \mathrm{C}$. Rate of reaction estimated from slope of initial voltage drop.

We also used absorbance measurements to monitor the kinetics of an enzyme reaction on the chip surface. Specifically, the sugar X-galactose (X-gal) was hydrolyzed into the products galactose and 4-Cl-3-Br indigo, using the enzyme beta-galactosidase ( $\beta$-gal) as a catalyst [18]. 4-Cl$3-\mathrm{Br}$ indigo is a deep blue precipitate [19], which strongly absorbs the $632.8 \mathrm{~nm}$ line of the $\mathrm{He}-\mathrm{Ne}$ laser. The microfluidic chip was programmed to merge one $400 \mathrm{nl}$ droplet of a phosphate buffer solution containing 1 unit of $\beta$-gal with another $400 \mathrm{nl}$ drop of $200 \mathrm{mM} \mathrm{X}$-gal in dimethylsulfoxide at $22{ }^{\circ} \mathrm{C}$. The photodetector response is 
shown in Fig. 6. The abrupt attenuation of the guided light, highlighted by the sloped dashed line, represents the initial reaction rate. During this period, the enzyme is primarily converting $\mathrm{X}$-gal to the precipitate. As more precipitate is produced, the enzyme also catalyzes the reverse reaction until equilibrium is attained and the attenuation is constant. While not shown here, preliminary studies indicate that a higher operating temperature will lead to an enhanced absorption rate as more precipitate is produced during the initial reaction. The initial notsy attenuation observed in the experiment is primarily caused by the optical effects incurred during positioning and mërging of the droplets over the beam path.

\section{CONCLUSION}

The studies presented here demonstrate the successful integration of evanescent wave sensing with thermocapillary actuation as a non-invasive sensor for droplet location and composition. Similar integration can be achieved with other surface tension driven microfluidic devices based on electrowetting-on-dielectric $[3,4]$ or dielectrophoretic pumping [5], which rely on open transport of droplets on the surface of glass or silicon wafers. Complete device integration is possible by replacing the prism couplers with grating couplers and combining the chip with solid-state light sources and detectors.

\section{ACKNOWLEDGEMENTS}

This work was funded by the NASA Microgravity Fluid Physics Program and the US Army TACOM-ARDEC. JPV wishes to thank Dr. P. K. Tien of Bell Labs for helpful discussions. SMT gratefully acknowledges the Moore Distinguished Scholar Program at Caltech, where she is currently on leave, for the generous bospitality and financial support extended during her stay.

\section{REFERENCES}

[1] A. A. Darhuber, J. P. Valentino, J. M. Davis, S. M. Troian and S. Wagner, "Microfluidic Actuation By Modulation of Surface Stresses", Appl. Phys. Lett. Vol. 82 pp. 657-659: Jan. 2003.

[2] A. A. Darhuber, J. P. Valentino, S. M. Troian and S. Wagner, "Thermocapillary Actuation of Droplets on Chemically Pattemed Surfaces by Programmable Microheater Arrays", J. Microelectromech. Syst. Vol. 12 pp. 873-879: Dec. 2003.

[3] J. Lee and C. J. Kim, "Surface-Tension-Driven Microactuation Based on Continuous Electrowetting", $J$. Microelectromech. Syst. Vol. 9 pp. 171-179: Jun. 2000.

[4] M. G. Pollack, R. B. Fair and A. D. Shenerov, "Electrowetting-Based Actuation of Liquid Droplets For Microfluidic Applications", Appl. Phys. Lett. Vol. 77 pp. 1725-1726: Sept. 2000.
[5] T. B. Jones, M. Gunji, M. Washizu and M. J. Feldman, "Dielectrophoretic Liquid Droplet Actuation and Nanodroplet Formation", J. Appl. Phys. Vol. 89 pp. 14411448: Jan. 2001.

[6] J. Z. Chen, A. A. Darhuber, S. M. Troian and S. Wagner; "Capacitive Sensing of Droplets For Microfluidic Devices Based On Thermocapillary Actuation", Lab Chip Vol. 4 pp. 473-480: Oct. 2004.

[7] H. Ren, R. B. Fair and M. G. Pollack, "Autontated OnChip Droplet Dispensing With Volume Control By Electrowetting Actuation and Capacitance Metering", Sens. Actuators B, Vol. 98 pp. 319-327: March 2004.

[8] T. E. Plowman, J. D. Durstchi, H, K. Wang, D. A. Christensen, J. N. Herron and W. M. Reichert, "MultipleAnalyte Fluoroimmunoassay Using an Integrated Optical Waveguide Sensor", Anal. Chem. Vol. 71 pp. 4344-4352: Oct. 1999.

[9] E. Verpoorte, "Chip Vision-Optics for Microchips", Lab Chip Vol, 3 pp. 42N-52N: Aug. 2003.

[10] K. Misiakos, S. E. Kakabakos, P. S. Petrou and H. H. Ruf, "A Monolithic Silicon Optoelectronic Transducer as A Real-Time Affinity Biosensor", Anal. Chem. Vol. 76 pp. 1366-1373: March 2004.

[11] R. Bernini, S. Campopiano, L. Zeni and P. M. Sarro, "ARROW Optical Waveguides Based Sensors", Sens.

Actuators B, Vol. 100 pp. 143-146: Feb. 2004.

[12] K. B. Mogensen, J. El-Ali, A. Wolff and J. P. Kutter, "Integration of Polymer Waveguides for Optical Detection In Microfabricated Chemical Analysis Systems", Appl. Opt. Vol. 42 pp. 4072-4079: July 2003.

[13] Z. Wang, J. El-Ali, M. Engelund, T. Gotsaed, I. R. Perch-Nielsen, K. B. Mogensen, D. Snakenborg, J. P. Kutter and A. Wolff, "Measurements of Scattered Light on a Microchip Flow Cytometer With Integrated Polymer Based Optical Elements", Lab Chip Vol. 4 pp. 372-377: Apr. 2004. [14] D. Marcuse, Theory of Dielectric Optical Waveguides, Academic Press, Inc., San Diego, CA, pp. 1-19: 1991. [15] S. S. Saavedra and W. M. Reichert, "Integrated Optical Attenuated Total Reflection Spectrometry of Aqueous Superstrates Using Prism-Coupled Polymer Waveguides", Appl. Spectrosc. Vol. 44 pp. 1210-1217: July 1990.

[16] D. A. Stephens and P. W. Bohn, "Prism Coupling Efficiencies By Direct Measurement of the Coupling Gap in Integrated Optical Structures", Appl. Optics, Vol. 25 pp. 2865-2868: Sept. 1986.

[17] R. Ulrich and R.Torge, "Measurement of Thin Film Parameters With a Prism Coupler", Appl. Opt. Vol. 12 pp. 2901-2908: Dec. 1973.

[18] J. Sambrook, Molecular Cloning: A Laboratory Manual $2^{\text {nd }}$ ed., Cold Spring Harbor Laboratory, Cold Spring Harbor, NY, pp. 230-231: 1989.

[19] G. R. MacGregor, G. P. Nolan, S. Fiering, M. Roederer and L. A. Herzenberg, "Substrates for Cytochemical Demonstration of Enzyme Activity. I. Some Substituted 3Indolyl- $\beta$-D-glycopyranosides", Vol. 7 pp. 574-575: July 1964. 